\title{
Fewer Hospital Visits for Acute Stroke and Acute Coronary Syndrome During the COVID-19 Pandemic: A Reality or a Myth?
}

\author{
Ali Seifia, d, Jared Alexander Stowers ${ }^{\mathrm{b}}$, Reza Behrouz ${ }^{\mathrm{c}}$
}

The first documented case of coronavirus disease 2019 (COVID-19) in the USA was reported in a 35-year-old man from Snohomish County, Washington on January 20, 2020. Since then, there have been almost 5,000,000 confirmed cases all around the world and more than 1,500,000 cases in the USA [1]. In spite of the unprecedented burden on healthcare infrastructure by this global pandemic, clinicians across the USA have made anecdotal observations in established health care trends. Specifically, the authors as well as other clinicians around the world have noted decreased hospital and emergency room visits and hospitalizations for acute stroke (AS) and acute myocardial infarction (AMI) at their institutions and the other hospitals around the world [2-9]. If this observation does indeed hold and is universal, reasons for it are manifold. Here, the authors present various theories for this observation, which is mostly based on the recently published opinions [2-9] and personal observation and communications with multiple clinicians who are involved in the care of AS and AMI patients.

One theory is that the presentation of AS and AMI to hospitals is falsely low, and the patients do not go to the hospitals because of fear from the COVID-19 pandemic [2]. Recently the European Stroke Organization (ESO) published a statement and mentioned that among 426 stroke care providers from 55 countries, only one in five reported that stroke patients are currently receiving the usual acute and post-acute care at their hospital [2]. Similarly, a preliminary analysis during the early phase of the COVID-19 pandemic showed an estimated 38\% reduction in the US cardiac catheterization for acute coronary syndrome (ACS), similar to the $40 \%$ reduction noticed in Spain $[5,6]$.

Fear and trepidation regarding exposure to the COVID-19

Manuscript submitted May 20, 2020, accepted May 27, 2020

Published online June 1, 2020

aNeuro Critical Care, University of Texas Health Science Center at San Antonio, San Antonio, TX 78229, USA

${ }^{b}$ School of Medicine, University of Texas Health Science Center at San Antonio, San Antonio, TX 78229, USA

${ }^{\mathrm{c} S}$ Stroke Program, Department of Neurology, University of Texas Health Science Center at San Antonio, San Antonio, TX 78229, USA

${ }^{\mathrm{d} C o r r e s p o n d i n g ~ A u t h o r: ~ A l i ~ S e i f i, ~ N e u r o ~ C r i t i c a l ~ C a r e, ~ U n i v e r s i t y ~ o f ~ T e x a s ~}$ Health Science Center at San Antonio, 7703 Floyd Curl Drive, San Antonio, TX 78229, USA. Email: seifi@uthscsa.edu

doi: https://doi.org/10.14740/jnr601 in clinical settings may compel many to avoid calling for help, and "ride the storm" at home instead. It is possible that patients, often those who are elderly and with multiple comorbidities, are deferring emergency services and are dying at home during this pandemic. A study in the UK reported a $25 \%$ decrease in emergency room admissions 1 week after initiation of the nationwide lockdown in March 2020 [10]. With the exceptions of admissions for pneumonia, which have increased, other morbidities such as myocardial ischemia and gastrointestinal abnormalities decreased [10].

Widespread stay-at-home mandates and city lockdowns have been adopted across the USA, and it could be another contributor to reduced emergency room visit rates for symptoms that are not typical of COVID-19. Some patients may even presume that hospitals and clinics are closed altogether, or doctors are too busy treating patients with COVID-19 to treat patients with stroke [2-4].

A study in 2019 found that individuals with intimate social networks consisting mainly of family members versus more robust social connections such as friends or co-workers were more likely to delay presentation for emergency services [11]. The study found that family members were more likely to opt for a watch-and-wait approach to symptom development, while non-family members were more likely to suggest seeking immediate medical attention. In a statement by the University of Texas Southwestern for the COVID-19 pandemic of patients isolated from loved ones, it is possible and perhaps likely that some stroke symptoms are going unnoticed [3].

In the USA, as social-isolation and self-quarantine become the norms, social networks are restricted, and, in many cases, people are limiting their exposure to their nuclear family. It is likely that patients are influenced by family to stay home, possibly out of fear of exposure to COVID-19 and passing it on to other family members. This temporal pattern could be similar to the "Christmas effect", where intake census of non-life-threatening events is low during significant societal events [12]. Therefore, patients experiencing symptoms of atypical angina or mild neurological deficits characteristic of a transient ischemic attack may opt to stay at home and monitor the symptom progression rather than seek care immediately. Authors of this article, however, argue that a more immediate social circle has benefited their patients, particularly those who struggle with medication adherence. The increased oversight from family members and less variable routines likely improved adherence to medications, 
and subsequently, better control of their chronic disease states that are risk factors for AS and AMI.

The Johns Hopkins Coronavirus Resource Center estimates that the mortality rate of COVID-19 in the USA is approximately $4 \%$ with a case fatality rate of about seven patients per 100,000 [1]. Many of the patients who die are those with substantial medical history. It can be suggested that patients who are dying from COVID-19 are the same ones who would present to emergency and inpatient services for acute events. Although the overlapping at-risk patient population is likely a contributing factor, it is doubtful that it is a sole contributor to our observation.

A beneficial, secondary consequence of the social distancing initiatives implemented across the country is the reduction of transmission of common respiratory pathogens such as influenza, in addition to mitigating the spread of COVID-19. The Centers for Disease Control Weekly US Influenza Surveillance Report indicates laboratory-positive influenza cases continue to decline, likely associated with social distancing measures and fewer in-person testing provider visits [13]. Influenza is welldocumented in literature to exacerbate pre-existing cardiac conditions and initiate life-threatening events such as non-STelevation myocardial infarction (NSTEMI), heart failure exacerbations, and arrhythmias [14]. With the implementation of public safety measures to reduce the incidence of new COVID-19 cases, there may be a conceivable decline in the prevalence of other respiratory diseases that tend to trigger cardiac and cerebrovascular events or exacerbate chronic conditions. Therefore, social distancing may represent an inadvertent protective factor.

Social distancing measures have resulted in drastic lifestyle changes for a significant number of Americans. As a large portion of the workforce is mandated to work from home, this might have an unexpected benefit. Eliminating ubiquitous stressors such as the daily commute to work, rush hour traffic, and stressful office settings has resulted in a catecholamine reduction and mental health improvements. On the contrary, the financial stress of an unpredictable economy, limited employment outlook, reduction of retirement securities, and overall social isolation may result in increased stress levels. A consensus is that although stress may be reduced overall in the short-term, long-term implications of this pandemic are dire.

The daily diets and physical activity levels of the typical Americans have changed significantly with the implementation of social distancing measures, as restaurants and gyms across the country have been forced to suspend their services. Home-prepared meals are more common, thus potentially reducing daily sodium consumption compared to dining in an establishment. Historically, there is a precedent for the suggestion that major shifts in diet due to a significant global event can impact the incidence and mortality of cardiovascular disease. A study in 1951 by researchers from the University of Oslo found that there was a marked decline in mortality from circulatory diseases in Norway from 1941 to 1943 - 1945, associated with drastic dietary changes due to World War II [15]. The researchers attributed a limitation in daily caloric intake with a shift away from cholesterol-laden foods as a reason for the stark reduction in incidence.

Individuals who are at particularly elevated risk of acute medical events, such as the elderly population, have fewer op- portunities for physical overexertion and induction of AMI or AS. Even alcohol consumption habits have changed, with alcohol sales in March 2020 increased by 55\% compared to March 2019 across the USA [16]. Despite reports of rampant binge drinking and increased alcohol sales throughout the country, people are drinking alcohol at home instead of at bars or nightclubs. Many practitioners have recognized a decreased in alcohol-related falls and motor vehicle accidents during the COVID-19 pandemic.

The paradoxical decrease in acute presentations of AS and AMI in the setting of the COVID-19 pandemic is perplexing, and it warrants further investigation by the medical community. Vast implications on the future of health care in the USA will be determined by the analyses of anecdotal observations such as those mentioned in this article. Further root cause analyses are warranted to quantify the extent of influence of medical adherence and lifestyle factors unique to the current COVID-19 pandemic experience to the observed deviations in incidence of AS and heart attack. This article highlights the importance of open communication between patients and providers. All physicians and health care providers must encourage their patients to seek emergency services or contact a physician if they develop any unusual medical symptoms, including those that are not typically associated with COVID-19.

\section{Acknowledgments}

None to declare.

\section{Financial Disclosure}

None to declare.

\section{Conflict of Interest}

None to declare.

\section{Author Contributions}

Ali Seifi was responsible for conceptualization, literature search, and writing the manuscript. Jared Stowers did the literature search and wrote the manuscript. Reza Behrouz edited the manuscript.

\section{Data Availability}

The authors declare that data supporting the findings of this study are available within the article.

\section{References}

1. Johns Hopkins Coronavirus Resource Center. https://coro- 
navirus.jhu.edu/. Accessed on May 19, 2020.

2. Van der Worp B. The European Stroke Organization (ESO) cautions against the consequences of the Coronavirus-Crisis on stroke care. https://eso-stroke.org/likely-increasein-the-risk-of-death-or-disability-from-stroke-during-thecovid-19-pandemic/. Accessed on May 19, 2020).

3. Welch B. Are stroke suffer silent victims of COVID-9? https://utswmed.org/medblog/covid19-stroke-volumedecrease/. Accessed on May 19, 2020.

4. Mcfarling UL, Where are all our patients? Covid phobia is keeping people with serious heart symptoms away from ERs. https://www.statnews.com/2020/04/23/coronavirusphobia-keeping-heart-patients-away-from-er/. Accessed on May 19, 2020.

5. Garcia S, Albaghdadi MS, Meraj PM, Schmidt C, Garberich R, Jaffer FA, Dixon S, et al. Reduction in ST-segment elevation cardiac catheterization laboratory activations in the United States during COVID-19 pandemic. J Am Coll Cardiol. 2020.

6. Oriol Rodriguez-Leor, Belen Cid-Alvarez, et al. Impact of the COVID-19 pandemic on care activity in interventional cardiology in Spain. REC Interv Cardiol. 2020.

7. Zhao J, Rudd A, Liu R. Challenges and potential solutions of stroke care during the coronavirus disease 2019 (COVID-19) outbreak. Stroke. 2020;51(5):1356-1357.

8. Mcnamara D. COVID-19: are acute stroke patients avoiding emergency care? https://www.the-hospitalist.org/hospitalist/article/220491/stroke/covid-19-are-acute-stroke- patients-avoiding-emergency-care. Accessed on May 19, 2020.

9. FOX news, ERs seeing less heart attacks, stroke patients during COVID-19. https://www.fox2detroit.com/news/ ers-seeing-less-heart-attack-stroke-patients-during-covid-19. Accessed on May 19, 2020.

10. Thornton J. Covid-19: A\&E visits in England fall by $25 \%$ in week after lockdown. BMJ. 2020;369:m1401.

11. Dhand A, Luke D, Lang C, Tsiaklides M, Feske S, Lee JM. Social networks and risk of delayed hospital arrival after acute stroke. Nat Commun. 2019;10(1):1206.

12. Walker NJ, Van Woerden HC, Kiparoglou V, Yang Y. Identifying seasonal and temporal trends in the pressures experienced by hospitals related to unscheduled care. BMC Health Serv Res. 2016;16:307.

13. Weekly U.S. Influenza Surveillance Report. Centers for disease control and prevention, centers for disease control and prevention. Accessed on April 10, 2020. https://www. cdc.gov/flu/weekly/index.htm.

14. Mamas MA, Fraser D, Neyses L. Cardiovascular manifestations associated with influenza virus infection. Int J Cardiol. 2008;130(3):304-309.

15. Strom A, Jensen RA. Mortality from circulatory diseases in Norway 1940-1945. Lancet. 1951;1(6647):126-129.

16. Polakovic Gary. COVID-19 drives alcohol sales and raises concerns about substance abuse. USC News, April 13, 2020. https://news.usc.edu/168549/covid-19-alcoholsales-abuse-stress-relapse-usc-experts/. 